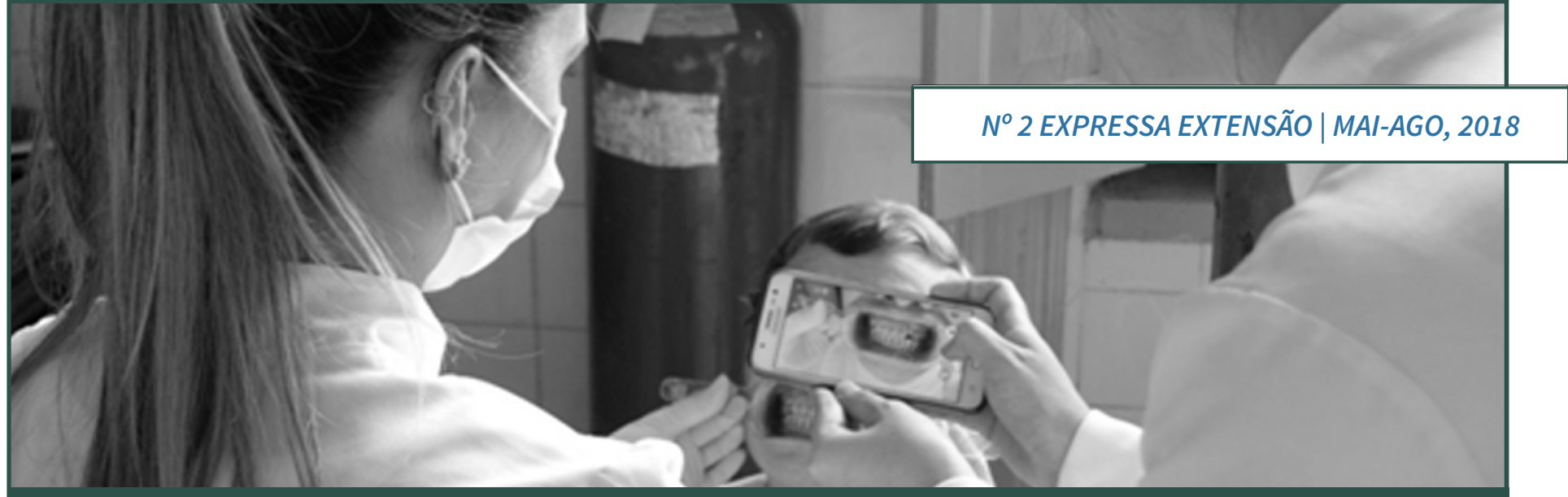

\title{
O OLHAR DO GEPETO E O CUIDADO COM A VIDA DE IDOSOS INSTITUCIONALIZADOS
}

THE LOOK OF GEPETO AND CARE FOR THE LIVES OF INSTITUTIONALIZED ELDERLY PEOPLE

Eduardo Dickie de Castilhos ${ }^{1}$ Maria Beatriz Junqueira de Camargo ${ }^{2}$ Tania Izabel Bighetti ${ }^{3}$

\section{RESUMO}

O projeto GEPETO - Gerontologia: Ensino, Pesquisa e Extensão no tratamento odontológico realiza as suas ações de extensão no Asilo de Mendigos de Pelotas. O objetivo deste trabalho é apresentar parte da memória visual do projeto relacionando com ações voltadas para a prevenção, tratamento e reabilitação que são desenvolvidas buscando qualidade de vida para os moradores. O registro fotográfico das práticas é realizado para ilustrar atividades de ensino e produção de pesquisa e extensão. A realização das fotos foi pactuada com a direção da instituição e é solicitada autorização verbal para todos os indivíduos antes do registro. As atividades acontecem um turno por semana onde se realiza: acolhimento de novos moradores, exames, tratamentos para alívio de dor, manutenção e restabelecimento de autonomia, tratamento de doenças bucais e reabilitação. Durante as atividades de extensão ocorrem trocas entre professores, acadêmicos e moradores. Além dos cuidados com a saúde, ocorrem discussões sobre espiritualidade, religiosidade e a finitude da vida. Assim, os cuidados com a saúde são encarados não como uma luta contra a morte, mas como uma forma de dar qualidade aos anos de vida compartilhados com os moradores.

Palavras-chave: Instituições de longa permanência para idosos. Assistência integral à saúde. Odontologia. Terapia ocupacional.

\footnotetext{
${ }^{1}$ Eduardo Dickie de Castilhos - UFPEL - Brasil - Possui graduação e mestrado em Odontologia pela Universidade Federal do Rio Grande e Doutorado pelo Programa de Epidemiologia da Universidade Federal de Pelotas. É professor adjunto da Universidade Federal de Pelotas. Tem experiência na área de Odontologia, com ênfase em Saúde Bucal Coletiva, e Odontogeriatria; eduardo.dickie@gmail.com; ${ }^{2}$ Maria Beatriz Junqueira de Camargo - UFPEL - Brasil - Possui graduação em Odontologia pela Universidade Estadual de Campinas. Especialista em Saúde Bucal Coletiva. Doutora em Epidemiologia pela Universidade Federal de Pelotas. Professora de Saúde Bucal Coletiva na Faculdade de Odontologia da Universidade Federal de Pelotas, biaj.camargo@gmail.com; ${ }^{3}$ Tania Izabel Bighetti - UFPEL - Brasil - Doutora em Saúde Pública pela Universidade de São Paulo. Professora associada nível I do Departamento de Odontologia Social e Preventiva da Faculdade de Odontologia, Unidade de Saúde Bucal Coletiva, na Universidade Federal de Pelotas, taniabighetti@ hotmail.com
} 


\section{ABSTRACT}

The project GEPETO - Gerontology: Teaching, Research and Extension in the dental treatment carries out its extension actions in the Asylum of Beggars of Pelotas. The actions are carried out jointly by academics of the courses of Dentistry and Occupational Therapy. The objective of this work is to present part of the visual memory of the project relating to actions aimed at prevention, treatment and rehabilitation that are developed seeking quality of life for the residents. The photographic record of the practices is carried out to illustrate activities of teaching and production of research and extension. The accomplishment of the photos was agreed with the direction of the institution and verbal authorization is requested for all the individuals before the registration. The activities take place once a week where activities are carried out such as: reception of new residents, examinations, treatments for pain relief, maintenance and restoration of autonomy, treatment of oral diseases and rehabilitation. Besides health care, there are discussions about spirituality, religiosity and the finitude of life. Thus health care is seen not as a struggle against death, but as a way of giving quality to the years of life shared with the residents.

Keywords: Homes for the aged. Comprehensive health care. Dentistry. Occupational therapy.

\section{INTRODUÇÃO}

A necessidade de mudanças na linha de cuidados à pessoa idosa tem sido alvo de muitas discussões. Neste sentido, destacam-se aspectos como a humanização do atendimento, bem como o fomento de inovações, através da disseminação de conhecimentos específicos para gestores e profissionais de saúde. Assim, é função das políticas de saúde contribuir para que mais pessoas alcancem idades avançadas com o melhor estado de saúde possível (BRASIL, 2010).

Com o envelhecimento populacional e a mudança no tamanho e conformação das famílias, a institucionalização de idosos passou a ser uma preocupação do poder público, de profissionais de saúde e de assistência social. As Instituições de Longa Permanência para Idosos (ILPI) surgiram fundamentadas pela caridade e no atendimento básico às necessidades da vida. As principais características das pessoas fragilizadas que buscam as ILPI são ausência ou precariedade de suporte familiar ou social; dificuldades financeiras; condições de saúde como sequelas de doenças crônicas, limitações físicas e cognitivas para as atividades da vida diárias, além de necessidade de acompanhamento após internações; opção pessoal (WATANABE; GIOVANNI, 2009). 
Estas características exigem uma atuação multiprofissional e interdisciplinar que é apontada nas Diretrizes Curriculares Nacionais de vários cursos, porém, nem sempre contemplada em seus projetos pedagógicos. Muitas vezes, a extensão universitária, pela sua característica de integrar ensino-pesquisa-extensão numa abordagem comunitária, acaba sendo um caminho para este tipo de atuação.

O objetivo deste trabalho é apresentar parte da memória visual do projeto relacionando com ações voltadas para a prevenção, tratamento e reabilitação que são desenvolvidas buscando qualidade de vida para os moradores do Asilo de Mendigos de Pelotas.

\section{DESENVOLVIMENTO}

O Asilo de Mendigos de Pelotas é uma ILPI filantrópica e foi fundado em 23 de setembro de 1882 (Fig. 1 e 2). Desenvolve cuidados permanentes para idosos com diferentes condições de vida e apesar do nome, atualmente atende idosos em condições socioeconômicas diversas. Os moradores apresentam diferentes motivos que levaram a institucionalização, como problemas de saúde, falta de suporte social ou por iniciativa própria visando reduzir a carga de tarefas decorrentes da vida sem companheiros e família. Além de serviços de hotelaria como refeições, cuidados com roupas e limpeza das áreas, conta com profissionais de saúde para administração de medicamentos, realização de curativos e outros cuidados frequentes para esse grupo de forma atender as suas necessidades individuais de saúde.

O projeto GEPETO - Gerontologia: Ensino, Pesquisa e Extensão no Tratamento Odontológico é desenvolvido no Asilo de Mendigos de Pelotas desde 2015 na modalidade de prestação de serviço. Tem como objetivo realizar atividades de atenção à saúde do idoso residente em instituições de longa permanência, com ênfase no tratamento odontológico. Conta com a participação de professores e acadêmicos dos cursos de Odontologia e Terapia Ocupacional. As ações desenvolvidas são assistência a idosos institucionalizados e atividade em laboratório de próteses. 
Figura 1 - Fachada do Asilo de Mendigos de Pelotas

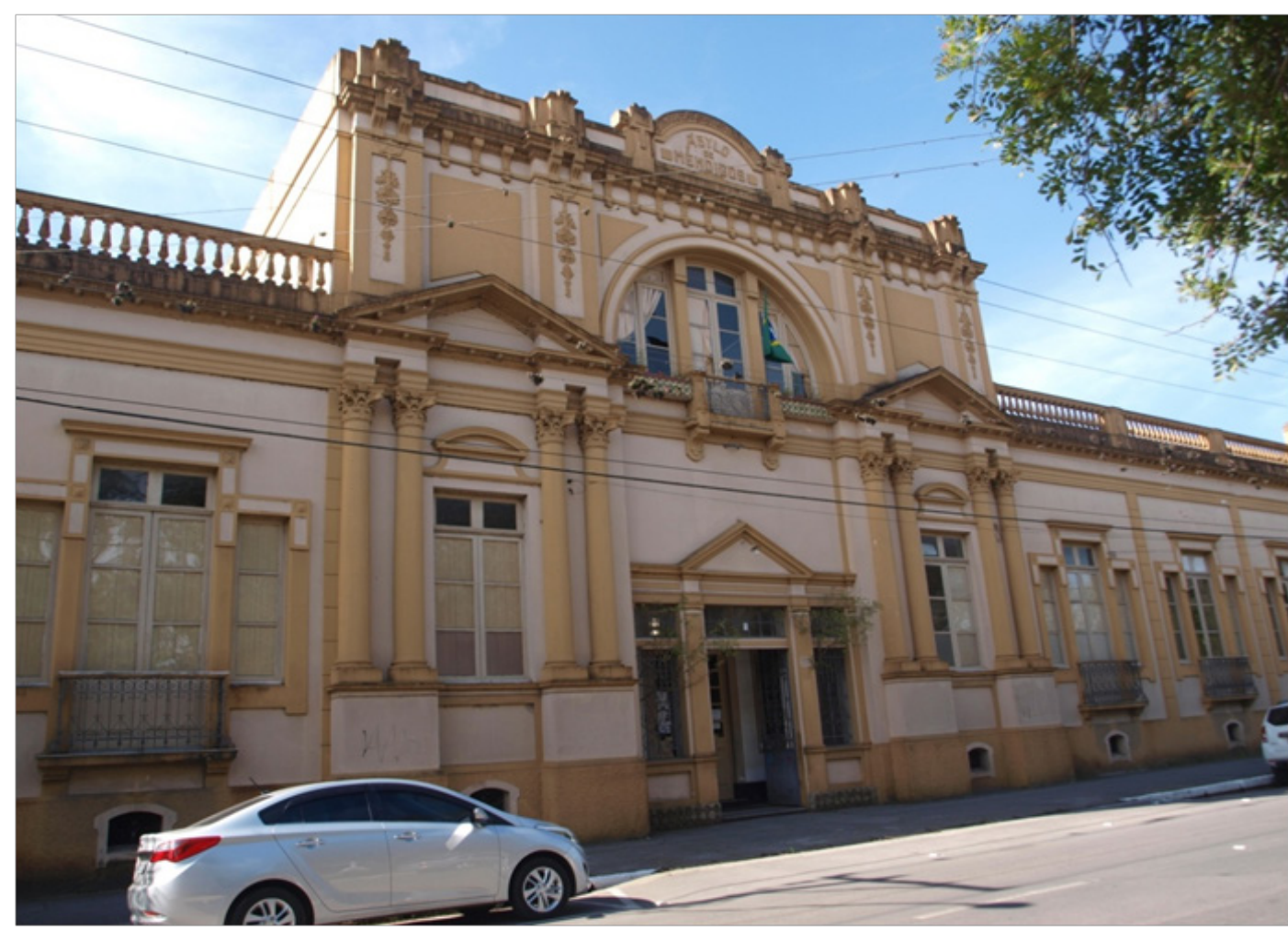

Fonte: autores

Figura 2 - Pátio interno do Asilo de Mendigos de Pelotas

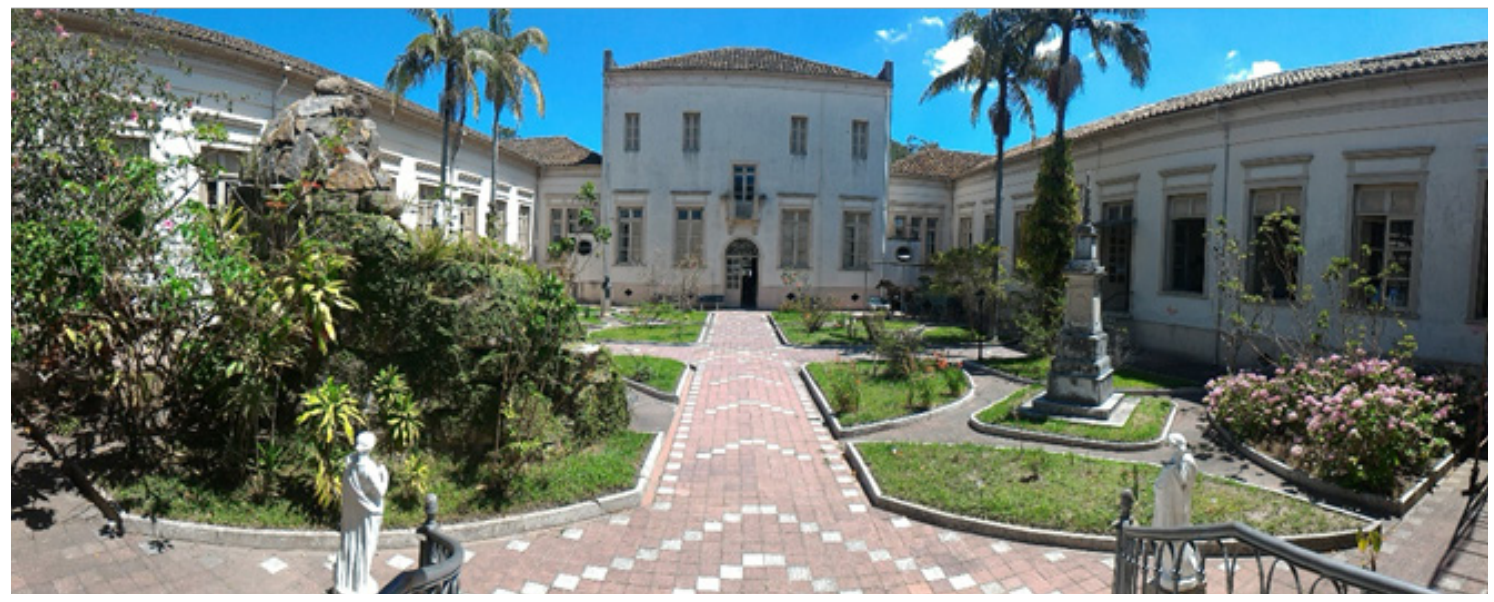

Fonte: autores 
Uma vez por semana os participantes do projeto se reúnem no Asilo e desenvolvem ações que vão de capacitações sobre assuntos como processo de envelhecimento, ações multiprofissionais e Gerontologia até assistência clínica e reabilitação (Fig. 3).

Figura 3 - Capacitação realizada no "claustro", onde residiam as freiras que atuavam no Asilo, sobre cuidados com moradores usuários de cadeiras de rodas

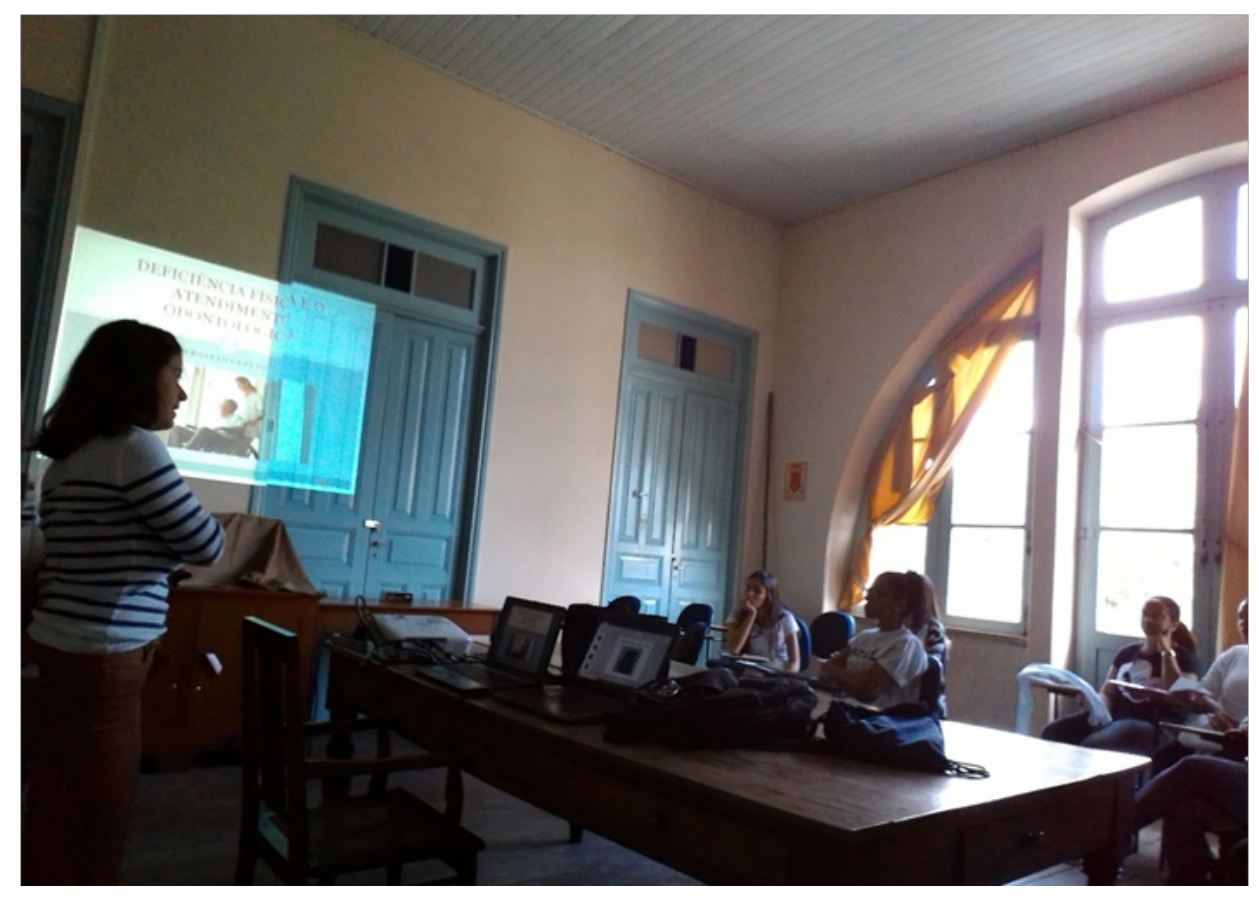

Fonte: autores

Todos os idosos da instituição são convidados a realizar um exame de saúde bucal. Aspectos gerais como motricidade, cognição e memória são abordados pela equipe de Terapia Ocupacional.

Quando se avaliam as demandas individuais, em função das características de cada morador, a atuação multiprofissional é muito importante. A parceria entre os cursos de Odontologia e Terapia Ocupacional propicia aos acadêmicos a oportunidade desta vivência, permitindo qualificar a formação dos futuros profissionais. Um exemplo é a interação dos acadêmicos para a orientação de morador com deficiência visual (Fig. 4). Outras ações envolvem questões cognitivas, memória e limitações motoras de membros inferiores e superiores. 
Figura 4 - Orientação de higiene bucal de morador cego em conjunto com acadêmicos da Odontologia e Terapia Ocupacional

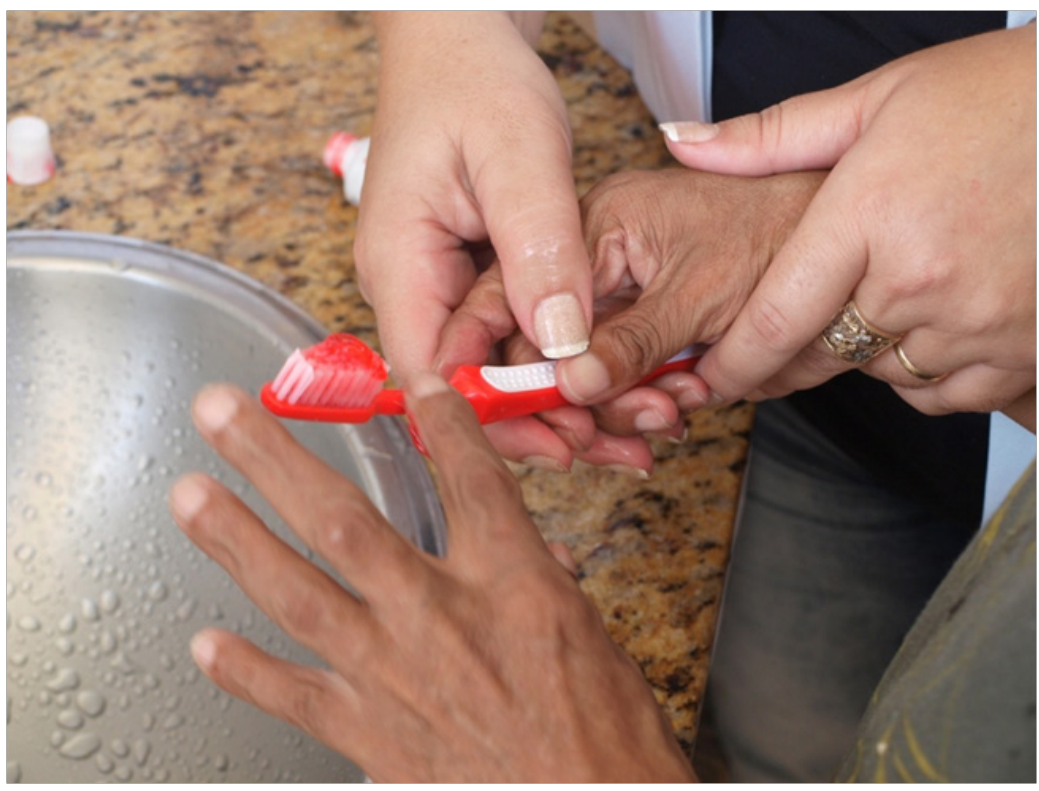

Fonte: autores

A integração dos acadêmicos dos dois cursos tem permitido momentos de reflexão e de troca de saberes. Isto pode ser evidenciado em momentos onde acadêmicos de Odontologia capacitam acadêmicos da Terapia Ocupacional para realizar orientações de higiene e identificar sinais e sintomas de problemas bucais enquanto estes demostram algumas formas de realizar a transferência de moradores da cadeira de rodas para a cadeira odontológica (Fig.5).

Figura 5 - Treinamento de acadêmicos de Odontologia para realizar a transferência de moradores de cadeira de rodas para cadeira odontológica após orientação de acadêmicos de Terapia Ocupacional

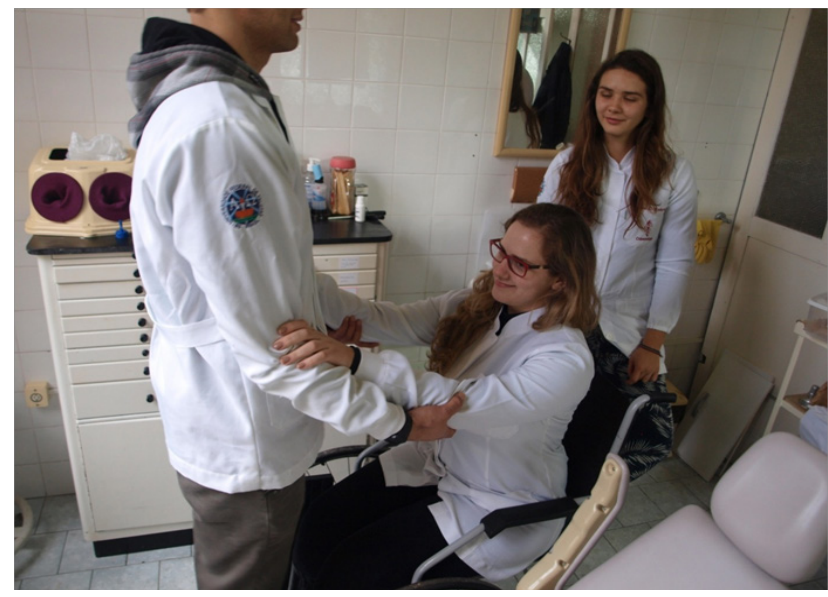

Fonte: autores 
No que diz respeito à saúde bucal, existe grande variedade de condições entre os idosos que podem favorecer ou comprometer a qualidade de vida. A presença de dentes naturais e crescente nessa população aumentando a demanda das necessidades dos moradores por prevenção e motivação (Fig.6). Estratégias como motivação e aplicação de verniz com flúor são importantes para a manutenção da saúde.

Figura 6 - Morador com a maioria dos dentes naturais com demandas relacionadas a prevenção e motivação

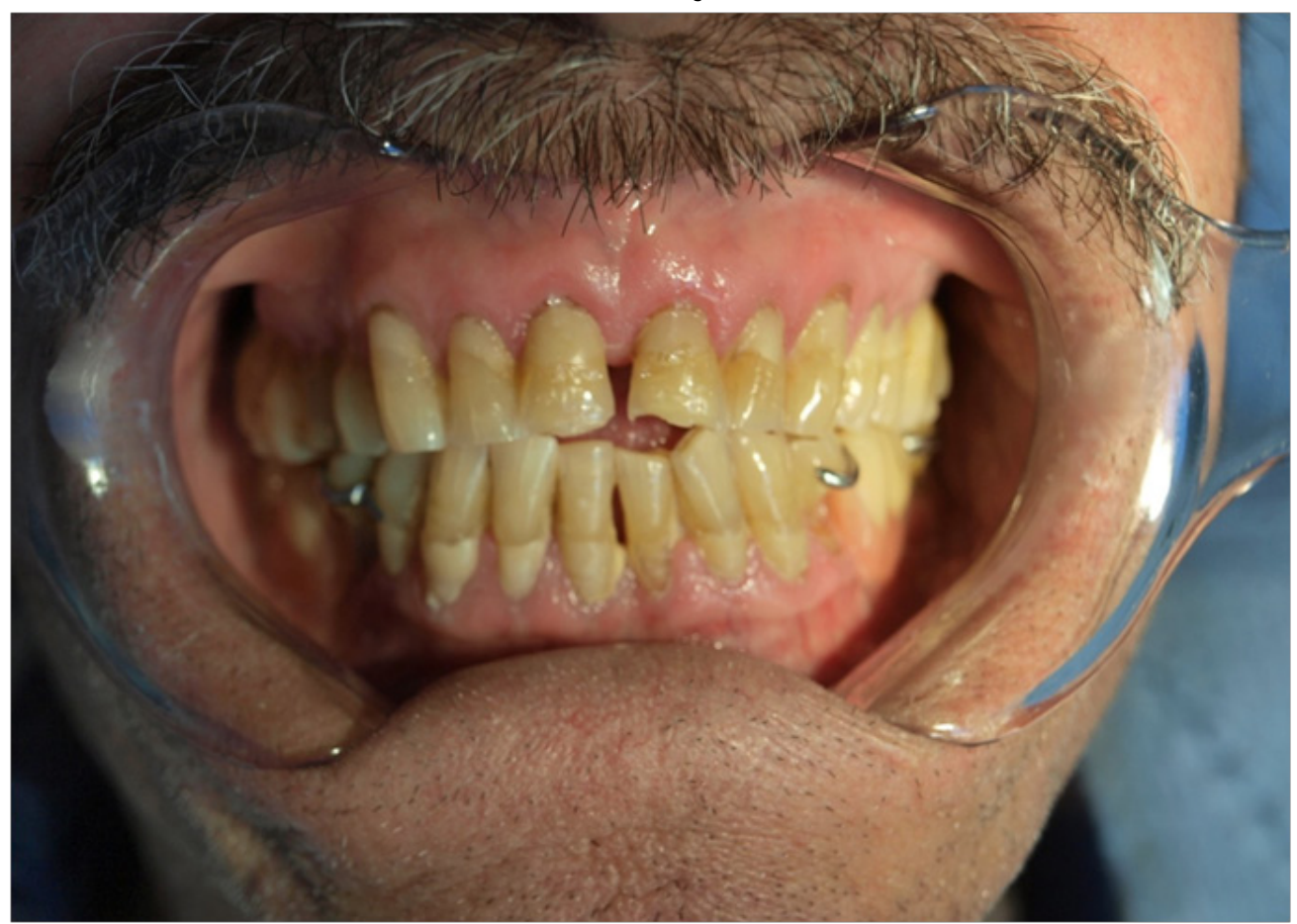

Fonte: autores

Além da necessidade de ações preventivas e motivacionais de saúde bucal, as demandas por tratamento e reabilitação em saúde bucal (Fig.7) são diversas. Isto requer capacitação dos acadêmicos para entender as mudanças próprias do envelhecimento que podem interferir nos tratamentos, bem como adequar os tratamentos buscando qualidade de vida mais do que a realização de tratamentos convencionais. Os idosos institucionalizados apresentam grande percentual de edentulismo,variando de 68 a $80 \%$ dependendo do arco (CARNEIRO, 2003; GRDEN, 2013; REIS, 2005; SILVA, 2008) e dentre esses em torno de $40 \%$ não usam próteses totais, superiores e inferiores, (CARNEIRO,2003). Entre os idosos dentados, as condições clínicas mais prevalentes foram cálculo (CARNEIRO, 2003) e dentes com necessidade de restauração e extração (GRDEN, 2013). Entre os moradores do Asilo $79,5 \%$ são desdentados totais e destes $75 \%$ usam próteses. 
Figura 7 - Morador após extrações dentárias com demandas restauradoras e necessidade de prótese dentária

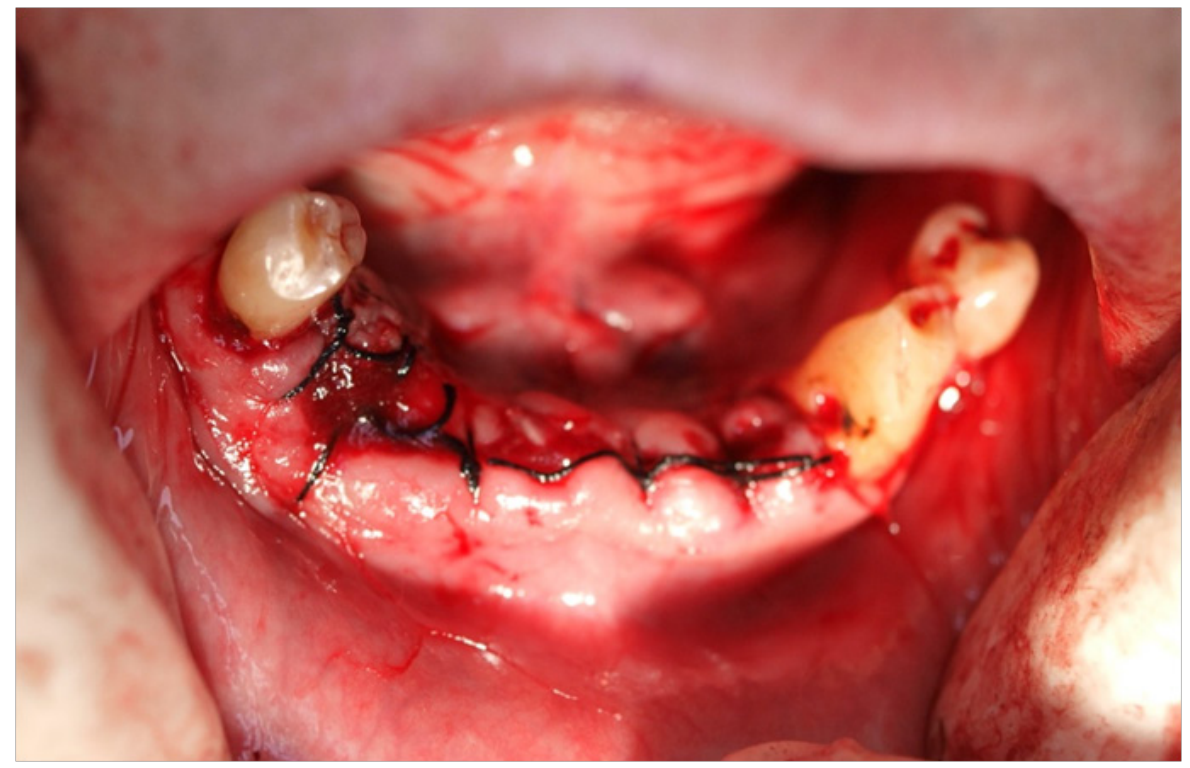

Fonte: autores

Por outro lado, moradores que já receberam algum tipo de reabilitação protética, trazem outras necessidades que vão exigir conhecimentos diversos. Estes vão do diagnóstico de condições inadequadas como desgaste (Fig. 8), fraturas e má condição de higiene. Os problemas mais frequentes entre os moradores são manchamento $(62,5 \%)$ e presença de cálculo $(71,9 \%)$.

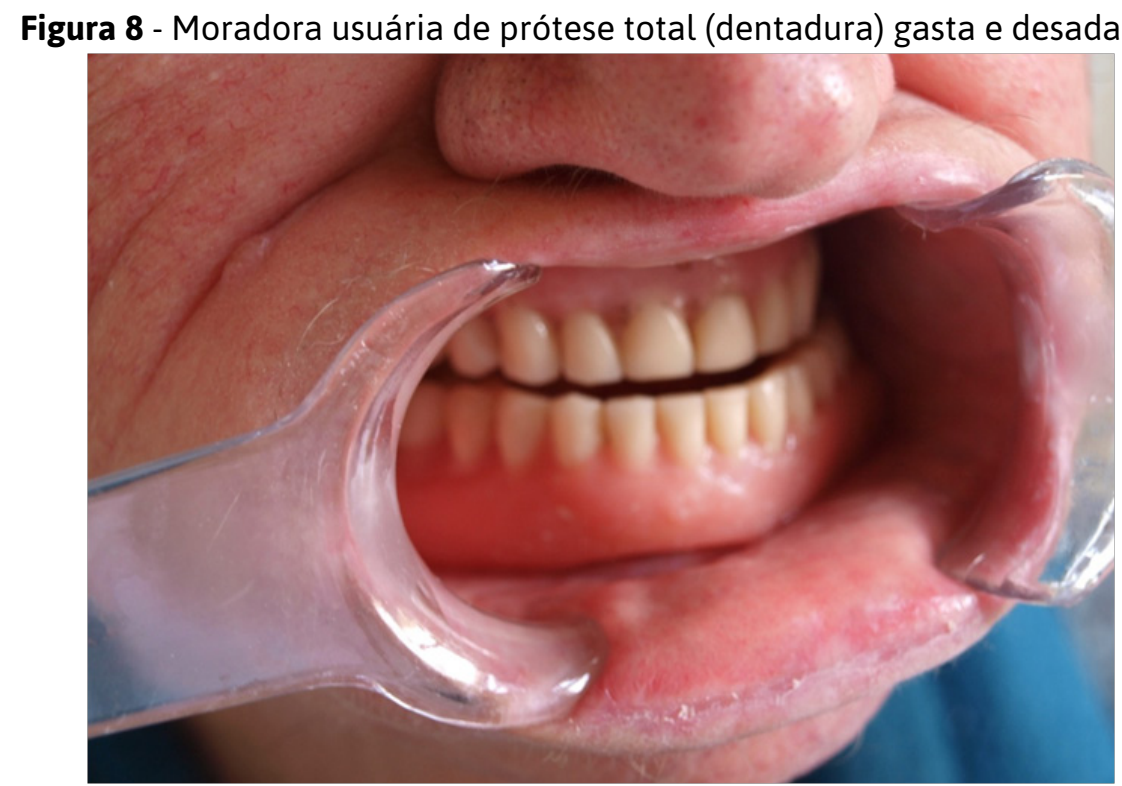

Fonte: autores

Atuar com este público na perspectiva da integralidade, exige do profis- 
sional de saúde o estabelecimento de vínculo e a compreensão de seus valores e crenças. Para isto, é importante incluir na assistência o sentido da espiritualidade, religiosidade e religião (Fig. 9) como recursos para a produção de saúde, valorizando sua história e o modo como o indivíduo se relaciona com a vida (CASTILHO; CARDOSO, 2015).

Figura 9 - Santa Apolônia (Padroeira da Odontologia) ao fundo e andador no primeiro plano. Relação entre aspectos físicos dos moradores e a espiritualidade presente no local

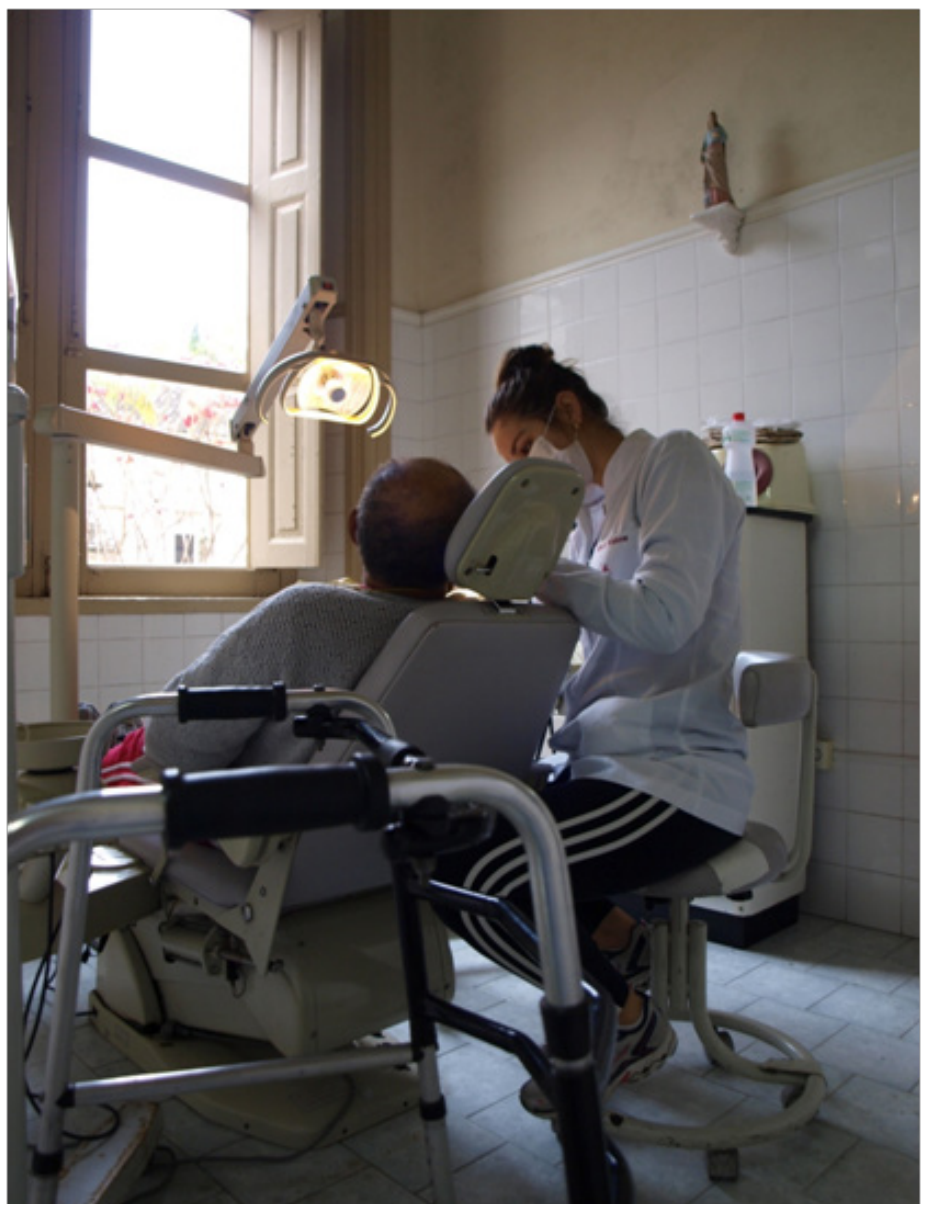

Fonte: autores

Apesar disso, uma característica sempre presente no cuidado com idosos é a questão da finitude humana. Para muitos profissionais a morte pode ser encarada como um fracasso pois a "missão" é combatê-la. Em se tratando de idosos institucionalizados, se deparar com a morte é algo mais frequente uma vez que eles apresentam pior condição de saúde geral e bucal quando comparados aos não institucionalizados. A preocupação em prolongar a vida é legítima, mas proporcionar mais "vida" nos dias que restam deve ser prioridade (REIS; MARCELO, 2006). Assim apesar das dificuldades em lidar com a morte, o projeto busca contribuir na formação de profissionais voltados para o cuidado com a vida de uma parcela da população carente de profissionais qualificados. 
Este trabalho procurou mostrar através de registros fotográficos que o projeto GEPETO atua na busca de qualidade de vida para uma população muito próxima da finitude. Esse projeto contribui na formação de profissionais mais humanizados e na produção de recursos (Fig. 10) que visam levar essa experiência para outros acadêmicos que não participam da ação de extensão. Os idosos da instituição se beneficiam tanto no aspecto humano quanto na questão de saúde.

Figura 10 - As ações de extensão além de beneficiar os idosos da instituição e acadêmicos envolvidos, servem de base para ações de pesquisa e ensino que podem ajudar na melhoria da qualidade de vida de outros idosos institucionalizados

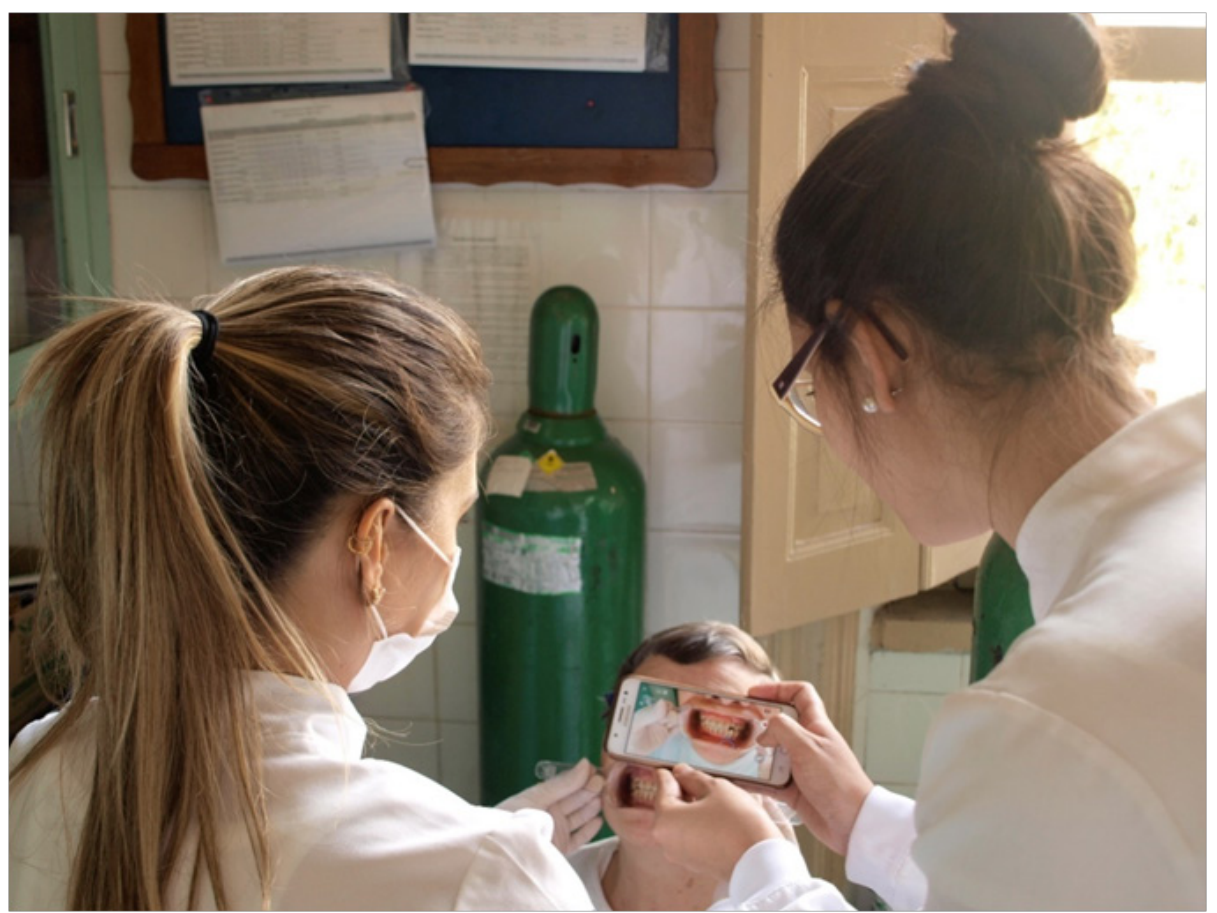

Fonte: autores

\section{REFERÊNCIAS}

BRASIL. Ministério da Saúde. Secretaria de Atenção à Saúde. Departamento de Ações Programáticas e Estratégicas. Área Técnica Saúde do Idoso. Atenção à saúde da pessoa idosa e envelhecimento. Brasília: Ministério da Saúde, 2010. 44 p.

CASTILHO,C. N.; CARDOSO, P. T.Espiritualidade, religiosidade e religião nas políticas públicas de saúde: um olhar para a integralidade. REFACS. v. 3, n. 1, p. 28-39, 2015.

CARNEIRO, R. M. V. et al. Saúde bucal de idosos institucionalizados, zona leste de São Paulo, Brasil, 1999. Cadernos de Saúde Pública.v. 21, n. 6, p. 1709-1716, 2005. 
GRDEN, C. R. B. et al. Avaliação da cavidade e higiene oral de idosas residentes em uma instituição de longa permanência. Cogitare Enferm. v. 18, n. 3, p. 490495 jul./set. 2013.

REIS, S. C. G. B. et al. Condição de saúde bucal de idosos institucionalizados em Goiânia-GO, 2003. Revista Brasileira Epidemiológica. v. 8, n. 1, p. 67-73, 2005.

REIS, S. C. G. B.; MARCELO, V. C. Saúde bucal na velhice: percepção dos idosos, Goiânia, 2005. Ciência e Saúde Coletiva. v. 111, n. 1, p. 191-199, 2006.

SILVA, S. O. et al. Saúde bucal do idoso institucionalizado em dois asilos de Passo Fundo - RS. RGO. v. 56. n. 3, p. 303-308, jul./set. 2008.

WATANABE, H. A. W. Instituições de Longa Permanência para Idosos (ILPI). Boletim do Instituto de Saúde, BIS, n. 47,p. 69-71, abr. 2009.

Data de recebimento: 05 de março de 2018.

Data de aceite para publicação: 03 de maio de 2018. 